\title{
Desarrollo de un dispositivo biomecatrónico adaptable al miembro superior humano para potenciación de ejercicios funcionales de acondicionamiento físico.
}

\author{
Sergio Franco Vargas, Javier Tovar Sánchez, Edgar Arcos Hurtado \& Leonardo Bermeo Varón \\ Universidad Santiago de Cali, Cali, Colombia. \\ sergio.franco01@usc.edu.co, javier.tovar00@usc.edu.co, edgar.arcos00@usc.edu.co, leonardo.bermeo00@usc.edu.co
}

Recibido: Julio 13, 2021.

Recibido en su versión corregida: Agosto 17, 2021.

Aceptación: Agosto 27, 2021.

https://doi.org/10.54606/Sextante2021.v25.02

Cómo citar: Franco Vargas, S., Tovar Sánchez, J., Arcos Hurtado, E. \& Pérez Bernal, M. (2021). Desarrollo de un dispositivo biomecatrónico adaptable al miembro superior humano para potenciación de ejercicios funcionales de acondicionamiento físico. Revista Sextante, 25, pp. $12-18,2021$.

\section{Resumen}

En este trabajo se presenta el desarrollo de un dispositivo biomecatrónico adaptable al miembro superior humano con doble funcionalidad, la primera es la de la prevención de enfermedades asociadas al sedentarismo a través de la potenciación de ejercicios funcionales de acondicionamiento físico, enfocándose principalmente en el fortalecimiento y musculación en las primeras fases posteriores a una lesión. La segunda es la de rehabilitación para personas con dificultades de movilidad en miembro superior, bien sea por causas naturales o accidentales, brindando en un sólo dispositivo portátil la capacidad de prevenir y rehabilitar enfermedades y/o lesiones del miembro superior. En el proceso del desarrollo del dispositivo se realizaron etapas de planificación, generación y evaluación de conceptos, diseño detallado, construcción, pruebas y ajustes, para finalmente llegar al prototipo que logró suplir de manera satisfactoria las fases de musculación y rehabilitación.

Palabras clave: Dispositivo biomecatrónico; Fortalecimiento de miembro superior; Rehabilitación de miembro superior.

\section{Development of a Biomechatronic Device Adaptable to the Human Upper Limb for Enhancement of Functional Exercises of Physical Conditioning.}

\begin{abstract}
In this work, the development of a biomechatronic device adaptable to the human upper limb with dual functionality is presented, first, prevention of diseases associated with sedentary lifestyle through the enhancement of functional conditioning exercises, mainly one hundred and fifty in the first phases after an exercise. Injury. Second, rehabilitation for people with mobility difficulties in the upper limb, either due to natural or accidental causes, providing in a portable device the ability to prevent and rehabilitate in prison and / or injuries to the upper limb. In the process of the development of the device, stages of planning, generation and evaluation of concepts, detailed design, construction, tests, and adjustments were carried out, to finally arrive at the prototype that managed to satisfactorily supply the muscle building phases.
\end{abstract}

Keywords: Biomechatronic device; Strengthening of upper limb; Upper limb rehabilitation. 


\section{Introducción}

La tecnología es un área importante en el desarrollo de dispositivos con múltiples objetivos, uno de esos objetivos es el campo de la rehabilitación, el cual busca, por medio de máquinas, realizar un trabajo de recuperación autónoma e independiente de una persona afectada en su sistema osteomuscular. Es así como el desarrollo tecnológico ha permitido construir dispositivos de rehabilitación, logrando incrementar la fuerza, movilidad y rendimiento del ser humano por medio de terapia asistida (Dellon \& Matsuoka, 2007).

Los dispositivos biomecatrónicos que permiten ejecutar movimientos programados en un miembro del cuerpo, en su mayoría están destinados al uso de las extremidades superiores e inferiores, estos tienen dos objetivos principales: la funcionalidad que permite realizar actividades cotidianas que la persona no puede hacer por sí misma, y la rehabilitación donde la persona realiza una recuperación de la parte afectada con el dispositivo. Según el DANE, gracias al registro de localización y caracterización de personas con discapacidad (Ministerio de Salud - RLPD-Colombia), se identifica que en Colombia hay 1'418.065 de personas inscritas, de las cuales el $34 \%$ (470.215) tiene un problema en la función motora del cuerpo debido una enfermedad o accidente, ocasionando que las personas afectadas tengan una dificultad en hacer labores cotidianas, problemas en su trabajo, en su vida personal y social. El 22\% (311.780) de las personas indicaron que necesitan rehabilitación fisioterapéutica y el $58 \%$ (180.197) no pudieron asistir a las terapias en el momento del registro. El $44 \%$ (257.421) de las personas refirieron que la causa de no asistir eran problemas económicos (Ministerio de Salud y Protección Social, 2018).

Es evidente que existe un problema (i), la no asistencia a las terapias en centro asistencial y (ii) problemas económicos, que, si bien no son claros los motivos, el porcentaje de la no realización de terapias de rehabilitación es bastante alto. Dentro de RLPD se incluyen las personas con patologías en los miembros superiores, la rehabilitación es realizada normalmente después del tiempo de inmovilización o de una intervención quirúrgica y con presencia de especialistas de la salud. Sin embargo, como ya se ha mencionado, un gran porcentaje de personas no pueden realizarse estas terapias a causa de problemas económicos o dificultades en los traslados a las instalaciones donde están los profesionales de rehabilitación. Adicionalmente, las entidades prestadoras del servicio de salud presentan problemas a la hora de agendar citas por falta de personal especialista o por sobrecarga en las terapias, una de las soluciones es el diseño de dispositivos y sistemas para dar solución a un amplio rango de necesidades que puedan asistir a la terapia en casa y permitan no sobrecargar las instituciones, logrando una mejora en el servicio de calidad y mayor cobertura. El dispositivo que se presenta en este artículo cuenta con dos aplicaciones: una de rehabilitación enfocada a ayudar a personas que han visto reducida su movilidad en miembro superior, bien sea por causa natural o accidental; por otra parte, el dispositivo se puede utilizar para potenciar la actividad muscular y así lograr el fortalecimiento de los músculos asociados a la flexión y extensión del codo. Objetivos similares a los presentados por Vitiello \& Roccella, (2012), Tiboni \& Faglia, (2018) y Pilla \& Trigili, (2020).

\section{Metodología}

Para el desarrollo del dispositivo biomecatrónico adaptable a miembro superior humano se utilizó una metodología exploratoria - investigativa conformada por diferentes etapas enmarcadas en un proceso de desarrollo, basadas en la generación de conceptos, como presentan Ulrich \& Eppinger, (2009).

\subsection{Planificación}

A partir de una etapa de planificación se identificaron necesidades a suplir en cuanto al fortalecimiento muscular, y a la rehabilitación del miembro superior, las cuales se listan en la Tabla 1.

\subsection{Generación de conceptos}

Durante esta etapa del proyecto se generaron conceptos para satisfacer los objetivos del dispositivo, ayudar al movimiento del miembro superior, y posterior a esto fortalecimiento muscular, para finalmente crear un diseño innovador con un punto de equilibrio que lograra una sinergia efectiva entre ambas funciones; los 
Tabla 1. Necesidades del dispositivo.

\begin{tabular}{llc}
\hline$\#$ & \multicolumn{1}{c}{ Necesidad } & Importancia \\
\hline 1 & $\begin{array}{l}\text { El dispositivo se adapta fácilmente } \\
\text { al miembro superior. }\end{array}$ & 5 \\
2 & $\begin{array}{l}\text { El dispositivo tiene un peso } \\
\text { cómodo. }\end{array}$ & 4 \\
3 & $\begin{array}{l}\text { El dispositivo permite potenciar } \\
\text { músculos del miembro superior. }\end{array}$ & 5 \\
4 & $\begin{array}{l}\text { El dispositivo permite rehabilitar } \\
\text { articulaciones del miembro superior. }\end{array}$ & 5 \\
5 & $\begin{array}{l}\text { El dispositivo tiene fácil } \\
\text { mantenimiento. }\end{array}$ \\
6 & $\begin{array}{l}\text { El dispositivo no causa daño al } \\
\text { usuario. }\end{array}$ \\
7 & $\begin{array}{l}\text { El dispositivo es portátil. } \\
8\end{array}$ & $\begin{array}{l}\text { El dispositivo se puede usar sin la } \\
\text { compañía de un profesional de la } \\
\text { salud. }\end{array}$ \\
9 & $\begin{array}{l}\text { El dispositivo se compone de partes } \\
\text { que se pueden remplazar fácilmente. }\end{array}$ & 3 \\
\hline
\end{tabular}

Fuente: Los autores.

siguientes conceptos fueron los más relevantes durante el desarrollo del diseño final del mismo.

\subsubsection{Concepto $N^{\circ} 1$}

Este diseño se enfoca en el objetivo de generar los movimientos del miembro superior derecho humano, cuenta con un motor DC de alta velocidad y bajo torque con el propósito de mover el exoesqueleto, una batería recargable de 5 voltios, un switch para encender y apagar el motor, y además hacerlo girar en sentido horario y antihorario, ayudando de esta forma a efectuar el movimiento flexor del miembro superior del usuario. Además, se agregaron leds al dispositivo que consumen poca corriente y no interfieren en el funcionamiento de este, simplemente dan estética al mismo tal como se aprecia en la Figura 1.

Ventajas: el diseño permite ayudar al movimiento flexor del miembro superior del usuario, bajo costo de producción, repuestos de fácil acceso. Desventajas: el motor implementado en este diseño no cuenta con el torque necesario para desplazar el exoesqueleto junto con el brazo del usuario, tampoco permite un control preciso lo cual fácilmente ocasiona un sobreesfuerzo al tensionar excesivamente el cable y sufrir recalentamiento, fracturar una pieza del dispositivo o finalmente ocasionar un daño en el miembro superior del usuario.

\subsubsection{Concepto $\mathrm{N}^{\circ} 2$}

El segundo concepto tiene el objetivo de oponer resistencia a los movimientos del miembro superior del usuario, usando una guaya que de forma conjunta con una mordaza ofrece una resistencia determinada al movimiento flexor del miembro superior del usuario, además, al agregar el brazo hidráulico permite aumentar o disminuir la resistencia mecánica tal como muestra la Figura 2.

Ventajas: El brazo hidráulico es una solución efectiva para implementar un sistema de resistencia a los movimientos del miembro superior del usuario de forma efectiva, especialmente en los movimientos que involucran al músculo encargado del movimiento flexor del miembro superior (bíceps).

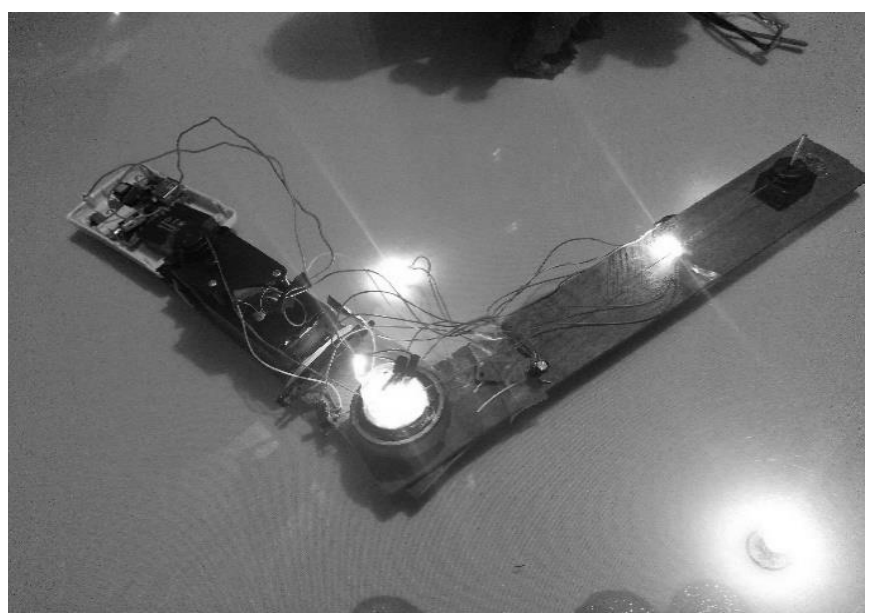

Figura 1. Concepto $\mathrm{N}^{\circ} 1$.

Fuente: Los autores.

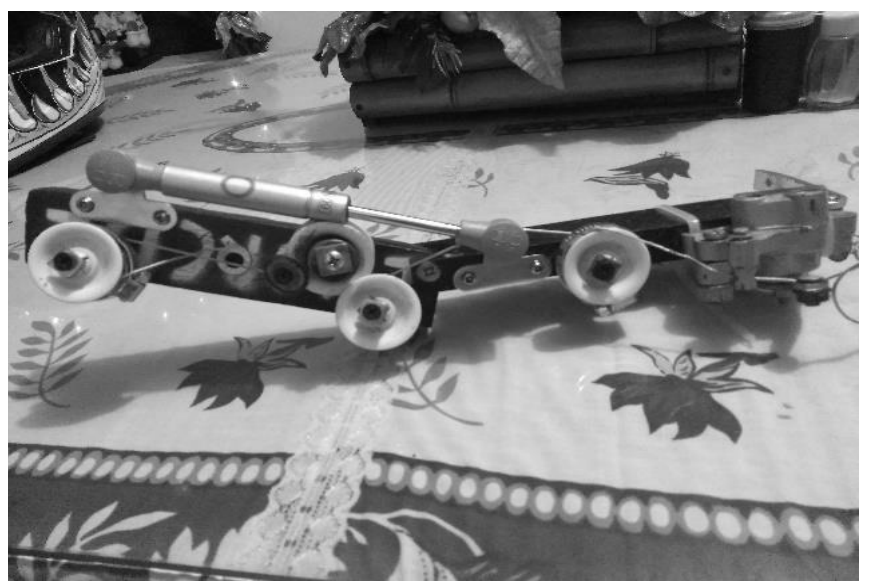

Figura 2. Concepto $\mathrm{N}^{\circ} 2$.

Fuente: Los autores. 
El brazo hidráulico puede intercambiarse, permitiendo que la resistencia se ajuste a la necesidad del usuario de acuerdo con su objetivo o su capacidad física, acompañando así al usuario en su proceso de ganancia de fuerza y masa muscular, sin dejar de ser útil en ninguna de sus etapas. Este diseño utiliza como mecanismo de resistencia una sola pieza, por lo cual se considera ideal para integrarlo y lograr una sinergia con un diseño con funcionalidad de ayuda al movimiento del miembro superior humano.

Desventajas: se determinó que el sistema de guayas y mordazas es innecesario en este diseño, pues este funciona de manera correcta en ausencia de estas piezas.

\subsubsection{Concepto $\mathrm{N}^{\circ} 3$}

En este concepto se tomó como referencia el conocimiento aquirido durante los diseños anteriores, siendo la base para este $\operatorname{los} \mathrm{N}^{\circ} 1$ y $\mathrm{N}^{\circ} 2$, creando un diseño (ver Figura 3), que logra una sinergia que se ve finalmente reflejada en la consecución de los objetivos del dispositivo, rehabilitación, y fortalecimiento.

Ventajas: el motor cuenta con una mejor precisión de movimiento y fácil control del exoesqueleto. Las carcasas tienen más superficie de contacto con el brazo del paciente, son cómodas y soportan los demás elementos del dispositivo, tales como el motor y el brazo hidráulico. El engranaje aumenta el torque del motor, generando un movimiento más suave y uniforme del dispositivo. Los brazos hidráulicos son intercambiables de acuerdo con la necesidad del paciente con su avance en el fortalecimiento muscular.

Desventajas: el peso del motor impide una sección larga en fase de rehabilitación. El material del dispositivo puede sufrir fracturas con un sobre esfuerzo.

\subsection{Diseño final}

El diseño final del dispositivo se presenta en la Figura 4, y en la Tabla 2. Se indican las partes y se mencionan las funciones que realiza cada una.

En la Tabla 2. Se presenta la función de cada elemento en el diseño final.
Tabla 2. Función de las partes del diseño final.

\begin{tabular}{ll}
\hline \multicolumn{1}{c}{ Elementos } & \multicolumn{1}{c}{ Función } \\
\hline Carcasa superior & $\begin{array}{l}\text { Brindar soporte al brazo del usuario y } \\
\text { el motor. }\end{array}$ \\
Pieza inferior & $\begin{array}{l}\text { Brindar soporte al antebrazo del } \\
\text { usuario, acompañar en el movimiento } \\
\text { flexo-extensor. }\end{array}$ \\
Motor. & $\begin{array}{l}\text { Mover el brazo del usuario. } \\
\text { Engranajes. }\end{array}$ \\
Brazo hidráulico. & $\begin{array}{l}\text { Oponer resistencia al movimiento del } \\
\text { usuario. } \\
\text { Tornillos }\end{array}$ \\
Base motor & $\begin{array}{l}\text { Asegurar o fijar el motor al } \\
\text { exoesqueleto. } \\
\text { Minimizar la friccion de las piezas }\end{array}$ \\
Balinera & superior e inferior. \\
\hline
\end{tabular}

Fuente: Los autores.

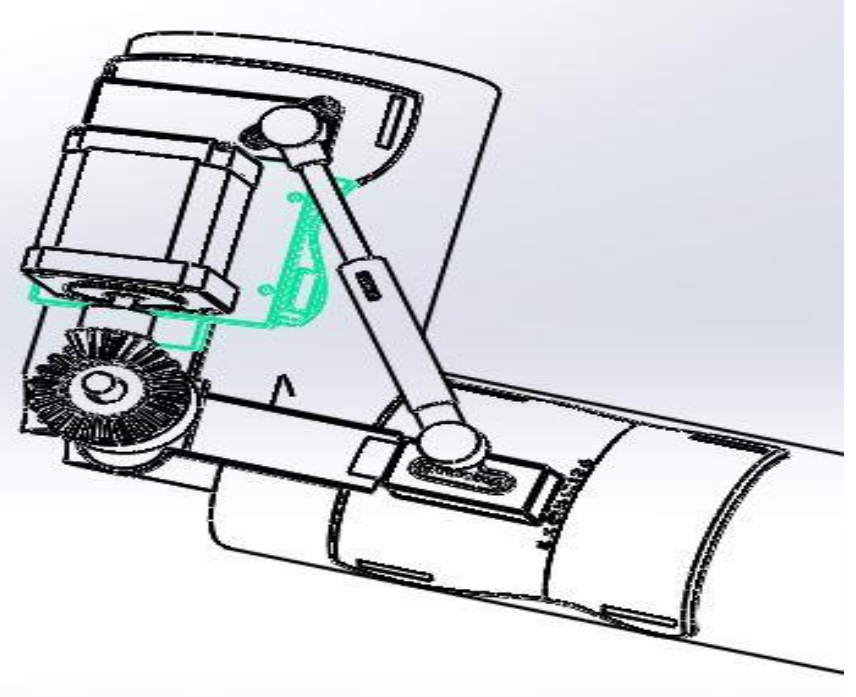

Figura 3. Concepto $\mathrm{N}^{\circ} 3$.

Fuente: Los autores.

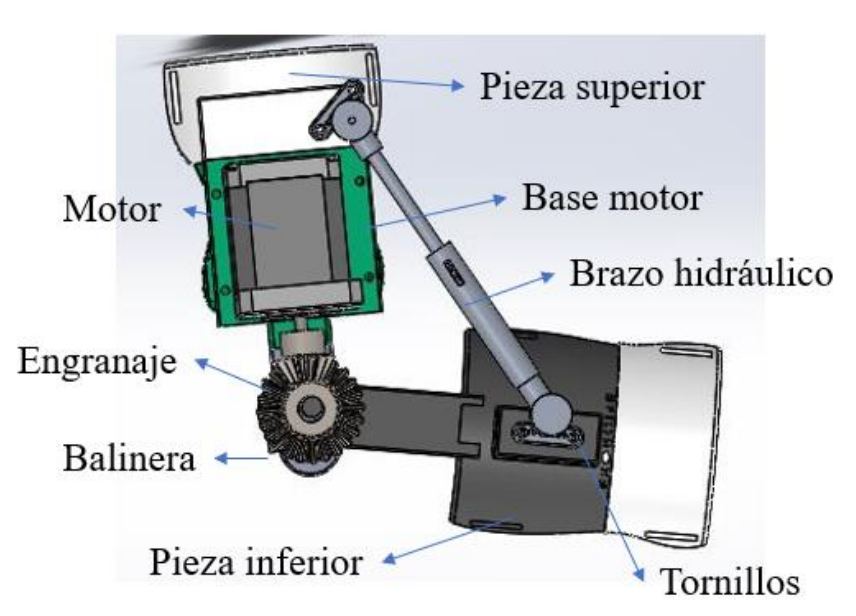

Figura 4. Diseño final.

Fuente: Los autores. 


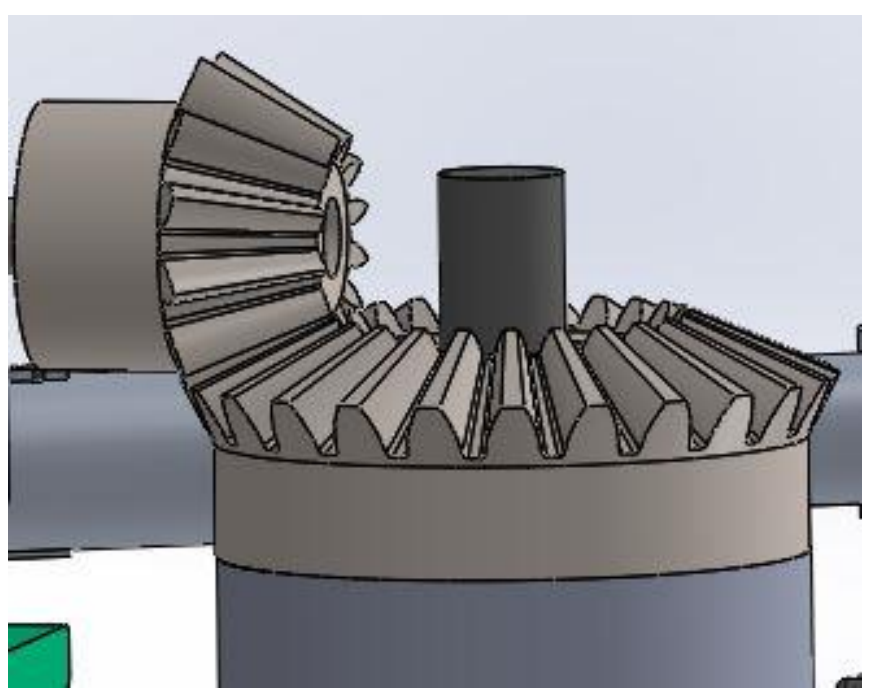

Figura 5. Engranajes cónicos rectos.

Fuente: Los autores.

\subsubsection{Selección del motor y engranaje}

Seleccionamos el motor paso a paso bipolar NEMA 23, que por sus características en precisión (200 pasos por vuelta, 1.8 grados cada paso) y (torque de $14 \mathrm{~kg} / \mathrm{cm}$ ) se determinó adecuado para este dispositivo, de acuerdo con el siguiente modelo matemático de flexión y extensión del codo, el cual representa una simplificación del modelo presentado por Mendoza \& Escudero (2007).

$$
\left(J+\frac{1}{4} M L^{2}\right) \ddot{\theta}+\frac{1}{2} M g L \operatorname{sen}(\theta)=T
$$

Donde:

$L=$ Longitud del antebrazo (valor promedio $)=28$ $\mathrm{cm}$.

$M=$ Masa del antebrazo (valor promedio) $=2 \mathrm{~kg}$.

$J=$ Momento de inercia en flexión y extensión del codo (valor promedio) $=0,243 \mathrm{~kg}$.

$\theta=$ Ángulo de la articulación del codo.

$T=$ Torque .

Los engranajes son importantes en los accionamientos de motores y maquinas; aumentan el par de salida, la velocidad y el sentido de giro (Myszka, D. H., 2004). Seleccionamos los engranajes cónicos que tienen como finalidad la transmisión del movimiento entre árboles que se cruzan formando un ángulo determinado, es decir, engranaje en forma de cono con dientes tallados en su superficie. La relación del par de salida para el dispositivo es 1:2 para aumentar el torque del motor y lograr desplazar el brazo del paciente. Ver Figura $\underline{5}$.

\subsubsection{Brazo hidráulico}

El brazo hidráulico le opone resistencia al movimiento flexo-extensor del codo del usuario para llevar una rehabilitación adecuada a cada persona de acuerdo con sus capacidades o necesidades en el proceso de rehabilitación; el brazo es intercambiable por otros de menor o mayor resistencia, medidas $88.72 \mathrm{~mm}$ de largo y $6.8 \mathrm{~mm}$ de ancho parte superior - $107 \mathrm{~mm}$ de largo y $14 \mathrm{~mm}$ de ancho parte inferior, el brazo hidráulico está compuesto por un material de aleación de aluminio.

\subsubsection{Sistema de control}

El sistema de control del sistema es de tipo ON/OFF, este permite controlar la articulación del codo en sus movimientos de flexión y extensión a través del motor y el sistema reductor. Se utilizó el controlador TB6600 de 4.0A basado en el chip TB6600 para controlar el motor, este es un módulo controlador de motor paso a paso de tipo bipolar que permite varios modos micro-paso, además es compatible con motores de 2 y 4 fases de $12 \mathrm{~V}$ a $40 \mathrm{~V}$. La corriente de excitación máxima es de menos de 5A. Mediante un Arduino uno se reciben las señales de los pulsadores (un pulsador para flexión, y otro para extensión) para procesarlas y enviarlas al controlador que controla los pulsos para accionar el motor.

\subsubsection{Ergonomía, simetría y material de construcción}

La estructura diseñada para el dispositivo se creó en forma de carcasa ajustable. La carcasa proporciona rigidez y resistencia estructural al dispositivo, y transfiere la carga de las extremidades humanas a los segmentos, el interior de las carcasas cubiertas con material tipo foamy está en contacto directo con el usuario y transfieren las cargas. Gracias al uso de un suave material ortopédico y una amplia área de interacción, contribuyen a reducir la presión sobre la piel del usuario y aseguran una interacción cómoda.

El material escogido para el diseño final fue el Acrilonitrilo butadieno estireno (ABS por sus siglas en inglés Acrylonitrile Butadiene Styrene), gracias 
a sus propiedades mecánicas, entre las que destacan su dureza, resistencia a químicos, estabilidad a altas temperaturas (vital cuando se trabajará con aparatos electrónicos), mantiene su tenacidad a bajas temperaturas (algunos plásticos tienden a volverse quebradizos en estas circunstancias) y por tales motivos el ABS suele ser un material muy usado en impresiones 3D.

Las piezas fueron diseñadas en un software CAD, lo cual garantiza precisión en cada una de sus medidas, y nos permite visualizar cómo se desempeña el dispositivo antes de ser construido y llevado a la práctica.

\section{Resultados}

En las Figuras $\underline{6}$ y $\underline{7}$ se puede ver el dispositivo construido siendo usado por personas con disminución de la capacidad motora en el miembro superior. En la Figura 6 puede verse el dispositivo siendo usado para rehabilitación del movimiento de flexión y extensión del codo. se puede ver que se ha omitido el brazo hidráulico, y se ha instalado el motor.

En la Figura 7 puede verse el uso del dispositivo, enfocado a potenciar los músculos que contribuyen al movimiento de flexión y extensión del codo, se puede ver que se ha omitido el motor, y se ha instalado el brazo hidráulico.

Se realizaron pruebas en una población de 20 personas, con el objetivo de conocer el desempeño del dispositivo en aspectos tales como: innovación, peso, comodidad y funcionalidad en cada una de sus fases, además de caracterizar a la población en su estilo de vida y conciencia de este. Los resultados obtenidos fueron los siguientes:

El $84 \%$ de la población considera que el dispositivo al utilizarse en fase de rehabilitación es pesado, lo cual se atribuye a la adición del motor, el $16 \%$ de la población considera que el dispositivo es liviano. El 12\% de la población considera que el dispositivo al utilizarse en fase de fortalecimiento es pesado, el $88 \%$ de la población considera que el dispositivo es liviano en esa fase. El $56 \%$ de la población considera que el dispositivo es poco cómodo para usar en fase de rehabilitación. El $66 \%$ de la población considera que el dispositivo es cómodo para usarlo en fase de fortalecimiento.

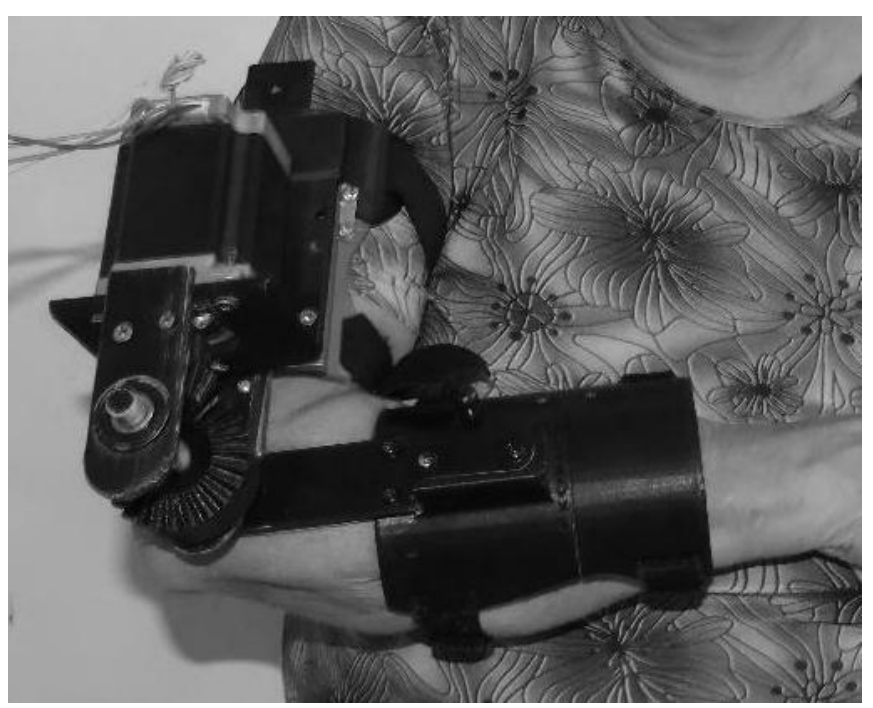

Figura 6. Uso del dispositivo para rehabilitación del codo. Fuente: Los autores.

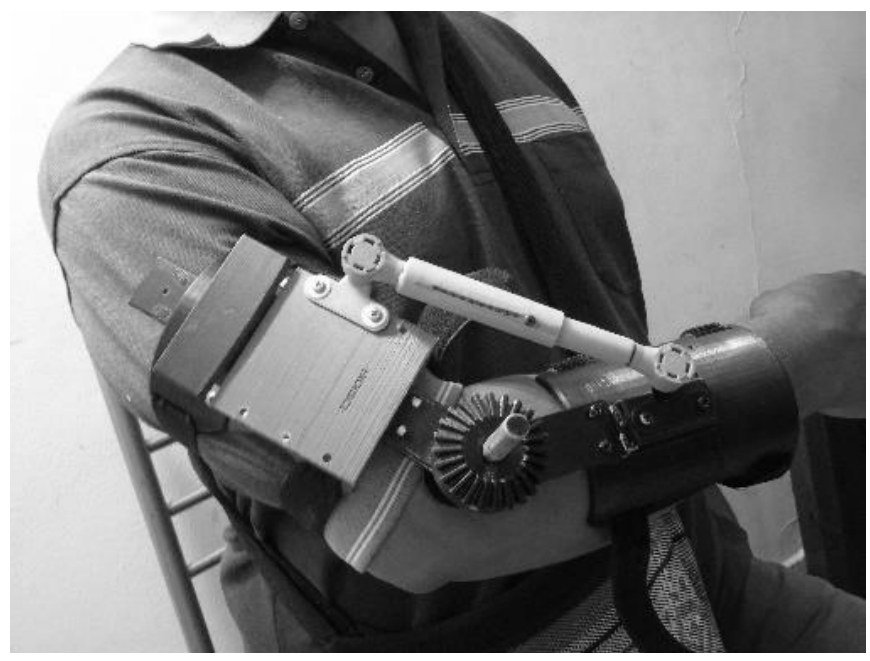

Figura 7. Uso del dispositivo para fortalecer músculos en movimiento de flexión de codo.

Fuente: Los autores.

El $100 \%$ de la población considera que el dispositivo cumple con las funciones de rehabilitación y fortalecimiento, aunque claramente manifiestan que debería mejorarse la comodidad.

\section{Conclusiones}

De acuerdo con los resultados obtenidos con el funcionamiento del dispositivo, se pudo evidenciar que el dispositivo logró conseguir de manera satisfactoria que las fases de musculación y rehabilitación cumpliesen sus objetivos. En fase de rehabilitación es importante trabajar con otro tipo de motores que proporcionen el torque necesario para realizar el movimiento de flexión-extensión, con una disminución en su tamaño y peso. El dispositivo es versátil, convirtiéndolo en un elemento de fácil manejo y adaptación al cuerpo humano en su fase de 
musculación/fortalecimiento. Debido a que los materiales utilizados para la elaboración del dispositivo son livianos, permite al usuario su fácil manipulación sin generar cansancio y molestia en fase de musculación/fortalecimiento. Es recomendable explorar otros materiales que brinden las comodidades descritas anteriormente, pero que sean aún más resistentes en un eventual uso permanente o casi continuo, no obstante que se ajuste a necesidades de usuarios que posean mayor masa corporal a fin de no causar incomodidades y ofrezca mayor resistencia.

El prototipo propuesto, el cual se encuentra en etapa exploratoria investigativa, nos brinda un punto de partida para proyectos futuros que tomando como base este diseño, puedan implementar exoesqueletos con doble funcionalidad para miembro superior $\mathrm{u}$ otros grupos musculares, dejando abierto un panorama para desarrollo con una inversión moderada, que logre tener un impacto a nivel personal, en salud pública, económico y social; beneficiando al usuario/paciente de forma continua y progresiva, además de brindarle la posibilidad de trasladar el dispositivo para continuar su proceso de rehabilitación, fortalecimiento o musculación en cualquier espacio.

\section{Referencias}

Dellon, B., \& Matsuoka, Y. (2007). Prosthetics, exoskeletons, and rehabilitation [grand challenges of robotics]. IEEE robotics \& automation magazine, 14(1), 30-34.

Registro de localización y caracterización de personas con discapacidad (Ministerio de Salud - RLPD-Colombia, 2019) https://www.minsalud.gov.co/proteccionsocial/p romocionsocial/Discapacidad/Paginas/registrolocalizacion.aspx

Ulrich, K. T., \& Eppinger, S. D. C. (2009). Diseño $y$ desarrollo de productos (No. 658.5752 Ul7d Ej. 1 022295). McGraw-Hill.

Mendoza-Vázquez, R., Escudero-Uribe, A. Z., \& Fernandez-Mulia, R. (2007, August). Simplified Analytical Dynamic Model for a Parallel Prosthetic Elbow. In 2007 29th Annual International Conference of the IEEE
Engineering in Medicine and Biology Society (pp. 3031-3034). IEEE.

Myszka, D. H. (2004). Machines and mechanisms. Prentice Hall.

Vitiello, N., Lenzi, T., Roccella, S., De Rossi, S. M. M., Cattin, E., Giovacchini, F., \& Carrozza, M. C. (2012). NEUROExos: A powered elbow exoskeleton for physical rehabilitation. IEEE transactions on robotics, 29(1), 220-235.

Tiboni, M., Borboni, A., Faglia, R., \& Pellegrini, N. (2018). Robotics rehabilitation of the elbow based on surface electromyography signals. Advances in Mechanical Engineering, 10(2), 1687814018754590.

Pilla, A., Trigili, E., McKinney, Z., Fanciullacci, C., Malasoma, C., Posteraro, F., ... \& Vitiello, N. (2020). Robotic rehabilitation and multimodal instrumented assessment of post-stroke elbow motor functions - a randomized controlled trial protocol. Frontiers in Neurology, 11, 1294. 